\title{
The implementation of the extended cohesive damage model for multicrack evolution in laminated composites
}

\author{
$\mathrm{X} \mathrm{Li}$ and $\mathrm{J}$ Chen* \\ School of Civil Engineering and Surveying, University of Portsmouth \\ Portland Street, Portsmouth PO1 3AH, UK
}

\begin{abstract}
In this work, the Extended Cohesive Damage Model (ECDM) was implemented to investigate the multicrack evolution in laminated composites. The ECDM was developed in terms of the framework of the extended fine element method (XFEM), but unlike the XFEM, the enriched effect to approximate existing crack together with the cohesive effect to approximate the crack tip behaviour was directly accounted into an equivalent stiffness matrix. A new damage scale relating to strain filed was introduced to account for delamination and matrix crack while the quasi-brittle failure mechanism was assumed for fibre fracture. The special mesh treatment for simulating interlaminar delamination required by conventional cohesive zone model $(\mathrm{CZM})$ is no longer required by the ECDM. Examples given in this paper proved the capability of the ECDM in capturing the multicrack evolution. This developed ECDM provides a novel modelling approach to investigate detailed multicrack failure mechanism in laminated composites.
\end{abstract}

Keywords: Extended cohesive damage model, Multicrack evolution, Laminated composites, Failure mechanism

Corresponding address: jiye.chen@ port.ac.uk 


\section{Introduction}

The damage mechanism in multidirectional fibre-reinforced composites features with various modes including transverse matrix crack, fiber fracture (under tension) or kinking (under compression), pulling-out of fiber from matrix, interlaminar delamination, debonding and fine-scale nonlinear shear deformation. Normally, multiple damage behaviour rather than single mode damage is observed within fibre composite structures and they often couple and interact with each other as a complex failure mode during damage propagation. The numerical prediction for this multiple damage procedure is significantly important in the design of composite structures. Many researchers made a lot of effort in the aspect of computational damage mechanics in the past two decades. However, effective and efficient predicting multicrack behaviour in laminated composite structures is still challenge.

Currently, vast majority of numerical research on computational modelling has been carried out to describe the progressive damage mode in laminated composites. Some existing numerical models are the approaches based on Continuum Damage Mechanics (CDM) originally developed by Kachanov [1] and Rabotnov [2]. The CDM approach characterizes the damage progress from virgin material up to structural collapse via degrading the stiffness of material during which the fracture is characterized as a smeared crack band that has the width of one element. The CDM has been widely used in the strength prediction of fiber composites [3-7]. Nevertheless, the reproducing ability for qualitative aspects of damage seems not 
particularly strong, i.e. detailed crack path in reality cannot be described by this approach, especially in the case of arbitrary crack propagation. Based on linear elastic fracture mechanics (LEFM), the virtual crack closure technique (VCCT) is able to effectively compute the crack propagation using a moving mesh technique. The main disadvantage of VCCT is the self-similar crack propagation requirement in advancing the crack front where local energy release rate reaching the threshold [8]. This makes VCCT hardly used in multiple damage modelling. The most widely-used approach to simulate delamination within the framework of finite elements is Cohesive Zone Model (CZM) [9]. In CZM, traction-separation law is normally applied within an interface element to characterize the nonlinearity in crack propagation, the validity of CZM has been well verified [10-12]. However, the numerical implementation of CZM is often through surface elements which need to be placed along the intended crack path. Furthermore, considerable efforts are obligated to ensure the element mesh exactly conforming to the potential crack interface, and the embedment of interface element is always cumbersome. When encountering multiple crack propagation by CZM, it would be hard to obtain a convergent solution.

Recently, a large number of publications reported modelling work on the arbitrary crack propagation without special treatment on FE mesh, among which, the eXtended Finite Element Method (XFEM) [13, 14] seems to be the most promising. The key feature in the XFEM formulation is the use of enrichment functions for cracked elements. This is achieved by enhancing the degrees of freedom (DoFs) of all the 
nodes employed by the elements with internal discontinuity. Since the extensive application of the XFEM, many improvements have been reported in the last decade. Fries et al. defined elements that blend the enriched areas with the rest of the domain where only standard finite elements were employed as 'blending element' [15]. It was recognised that these blending elements often require a special treatment in order to avoid a decrease in the overall convergent rate which was referred as a suboptimal convergent rate due to problems in blending elements [16]. The enhanced strain techniques or p-refinement were employed in blending elements to address this deficiency in their work [17]. Fries et al. [15] modified the enrichment functions such that they were zero in the standard elements, unchanged in the elements with all their nodes being enriched, and varied continuously in the blending elements. In so doing, there were no unwanted terms in the blending elements while the continuity of local enrichment functions was still maintained. This modification has assisted the XFEM to achieve optimal convergence and significantly improved the numerical performance. By the means of increasing the polynomial order of the approximation in only the blending elements, Tarancon et al. [18] enhanced the interpolation of the blending element on purpose of getting rid of the pathological terms in the approximation space of the blending element. Their numerical result has confirmed that the enhanced blending element always results in greater accuracy as well as convergent rate. A statically admissible stress recovery (SAR) scheme was introduced by Xiao et al. [19] to improve the accuracy of crack tip fields. Moreover, some 
important parameters controlling the accuracy of crack tip fields using the XFEM and the SAR were also discussed. Currently, the XFEM is available in a commercial FEM software ABAQUS. An overview of the resent development in the XFEM was given by Fries and Belytschko [20]. The XFEM is very effective for treating cracks or even multiple cracks in homogeneous materials, for which the enrichment functions are known. However, for complex heterogeneous material systems, such as laminated or textile composites, the enrichment functions are not readily available except for some very special cases such as a delamination crack at a symmetric plane in that case the bonded plate/beam can be treated as orthotropic materials and the singular stress field is known. Furthermore, the enrichments will lead to the modelling with more computationally expensive burden, which is repugnant for engineering application. Considering above limitations of the XFEM, Chen, et al previously investigated the basic concept of combing the XFEM with the CDM without using a specified enriched item to cope with the singularity problem at the crack-tip [29].

This paper presented a novel numerical approach, Extended Cohesive Damage Model (ECDM) to capture discontinuous fields resulting multicrack propagation, which was developed by combining the CZM and the XFEM and then eliminating the enriched DoFs. The ECDM formulation is a lower order equilibrium equation comparing to the standard XFEM, which permits nodal displacement calculation of the cracked element using standard FEM DoFs only. The micromechanical CZM was implicitly embedded within the model to describe the crack-tip singularity. The ECDM just uses standard 
finite element (FE) shape functions only, which enable the ECDM be compatible with standard FE programs. This paper firstly presented the basic ECDM formulation then focused on the numerical implementation as a user element via subroutine UEL in ABAQUS [21]. Different criterions and post-failure softening properties were introduced to address the characterization of multiple failure mechanisms in laminated composites. The sub-division integration schemes adopted by the ECDM user element were presented in this paper. Applications of the ECDM in modelling the multiple layered delamination in a laminated composite $\mathrm{T}$-joint and a stiffened laminated composite panel were carried out by this investigation. Detailed multicrack failure mechanism of the investigated composite examples was also given in this paper. These examples proved the capability and effectiveness of the implemented ECDM.

\section{Basic formulation of the ECDM}

Considering a solid body with a cohesive crack $\Gamma_{d}$ shown in Fig. 1, then the discontinuous boundary is a cohesive crack boundary $\Gamma_{\mathrm{d}}$. The displacement field was used in the ECDM with enrichments is given by Equ. 1.

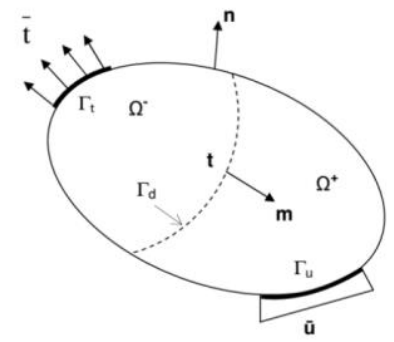

Fig. 1 Notation for a 2D domain with a cohesive discontinuity $\Gamma_{d}$

$$
\mathbf{u}^{h}(\mathbf{x})=\sum_{i \in I} N_{i}(\mathbf{x}) \mathbf{u}_{i}+\sum_{i \in J} N_{j}(\mathbf{x})\left(\mathrm{H}_{\Gamma_{d}}(\mathbf{x})-\mathrm{H}_{\Gamma_{d}}\left(\mathbf{x}_{i}\right)\right) \mathbf{a}_{i}
$$


Where, $\mathbf{x}_{i}$ is the position coordinate for the $i_{t h}$ node. $N_{i}$ is the conventional FEM shape functions associated with node $i . \mathbf{u}_{i}$ and $\mathbf{a}_{i}$ are the nodal variables associated with standard degree freedoms and enriched degree freedoms respectively at node $i$ and node $j$. The Heaviside step function $\mathrm{H}_{\Gamma_{d}}$ shown in Equ. 1 can be expressed by Equ. 2 for characterizing the physical jump when material completely separates.

$$
\mathrm{H}_{\Gamma_{d}}(\mathbf{x})= \begin{cases}1 & \mathbf{x} \in \Omega^{+} \\ 0 & \mathbf{x} \in \Omega^{-}\end{cases}
$$

Where, $\Omega^{+}$is the one side domain of discontinuity, the $\Omega^{-}$is another side domain. Using the Bubnov-Galerkin method, the trial functions and test function are chosen the same linear combination of interpolation functions as shown in Equ. 1. The discrete form of equilibrium equation for static analysis can be written as Equ. 3.

$$
\left[\begin{array}{ll}
\mathbf{K}^{u u} & \mathbf{K}^{u a} \\
\mathbf{K}^{a u} & \mathbf{K}^{a a}
\end{array}\right]\left[\begin{array}{l}
\mathbf{u} \\
\mathbf{a}
\end{array}\right]=\left[\begin{array}{l}
\mathbf{f}_{\text {ext }}^{u} \\
\mathbf{f}_{\text {ext }}^{a}
\end{array}\right]
$$

Where, $\mathbf{K}^{u u}, \mathbf{K}^{a a}$ and $\mathbf{K}^{u a}$ are the stiffness matrix associated with the standard FE approximation, the enriched approximation and the coupling between the standard FE approximation and the enriched approximation. $\mathbf{f}_{e x t}^{\mathbf{u}}$ and $\mathbf{f}_{e x t}^{a}$ are the equivalent nodal force vectors, $\mathbf{f}_{e x t}^{u}$ is for standard FEM freedoms while $\mathbf{f}_{e x t}^{a}$ is for enriched freedoms. In Equ. 3, the equivalent nodal force vectors without body force can be expressed as:

$$
\begin{aligned}
\mathbf{f}_{e x t}^{u} & =\int_{\Gamma^{h}} \mathbf{N}^{T} \overline{\mathbf{t}} d \Gamma \\
\mathbf{f}_{\text {ext }}^{a} & =\int_{\Gamma^{h}} \mathbf{N}^{T}\left(\mathrm{H}_{\Gamma_{\text {coh }}^{\text {crack }}}(x, y)-\mathrm{H}_{\Gamma_{\text {coh }}^{\text {crack }}}\left(x_{i}, y_{i}\right)\right) \overline{\mathbf{t}} d \Gamma+\mathbf{f}_{c o h}
\end{aligned}
$$


Where, $\overline{\mathbf{t}}$ is the external nodal force vector as shown in Fig. 1. The internal nodal force vector due to cohesive force $\mathbf{t}$ on the crack surface $\Gamma_{\text {coh }}^{\text {crack }}$ can be expressed as:

$$
\begin{aligned}
\mathbf{f}_{\text {coh }} & =\int_{\Gamma_{\Gamma_{\text {coh }}}^{\text {cract }}}\left(\mathrm{H}_{\Gamma_{d}}(x, y)-\mathrm{H}_{\Gamma_{d}}\left(x_{i}, y_{i}\right)\right) \mathbf{N}^{T} \mathbf{t} d \Gamma+\int_{\Gamma_{\text {conh }}^{\text {crack }}}\left(\mathrm{H}_{\Gamma_{d}}(x, y)-\mathrm{H}_{\Gamma_{d}}\left(x_{i}, y_{i}\right)\right) \mathbf{N}^{T}(-\mathbf{t}) d \Gamma \\
& =\int_{\Gamma_{\text {coh }}^{\text {crack }}} \mathbf{N}^{T} \mathbf{t} d \Gamma
\end{aligned}
$$

When the damage increases, the cohesive traction is assumed to decreases through a linear softening damage law shown in Fig. 2. Thus the traction $\mathbf{t}$ can be expressed as a function of initial traction and a damage scale $d$, i.e., $\mathbf{t}=\mathbf{t}_{\mathbf{0}} *(1-\mathrm{d})$. It should be noted that there is not a physical relative displacement jump $\Delta \delta$ before a crack formed.

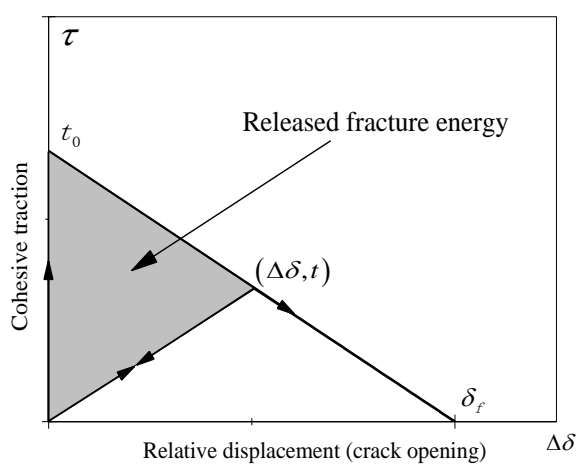

Fig. 2 A linear softening damage law

With the purpose of reaching a fully condensed equilibrium system, we eliminate the additional enrichment term a, thus the equilibrium equation with the standard FEM unknown quantities can be consequently obtained as shown in Equ. 6.

$$
\left(\mathbf{K}^{u u}-\mathbf{K}^{u a}\left(\mathbf{K}^{a a}\right)^{-1} \mathbf{K}^{a u}\right) \mathbf{u}=\mathbf{f}_{e x t}^{u}-\mathbf{K}^{u a}\left(\mathbf{K}^{a a}\right)^{-1} \mathbf{f}_{e x t}^{a}-\mathbf{K}^{u a}\left(\mathbf{K}^{a a}\right)^{-1} \mathbf{f}_{c o h}
$$

Considering practical engineering problems, we suppose there is no distributed external load applied on the cracked element. Then, the evolved equilibrium equations with standard FEM degree freedoms can be simplified as shown in Equ. 7. 


$$
\left(\mathbf{K}^{u u}-\mathbf{K}^{u a}\left(\mathbf{K}^{a a}\right)^{-1} \mathbf{K}^{u a}\right) \mathbf{u}=\mathbf{f}_{e x t}^{u}-\mathbf{f}_{c o h}^{\mathrm{int}}
$$

In Equ. 7, the calculation of the equivalent nodal force due to the existence of internal cohesive segment at a cracked element was given by Equ. 8 .

$$
\mathbf{f}_{c o h}^{\mathrm{int}}=\mathbf{K}^{u a}\left(\mathbf{K}^{a a}\right)^{-1} \mathbf{f}_{c o h}
$$

Normally, the released fracture energy as shown in Fig. 2 should be used to describe the crack propagation. In the proposed ECDM, an equivalent damage scale was introduced to avoid the appearance of the enriched DoFs related displacement gap $\Delta \delta$. The strain energy released due to the fracture should be equal to the released work done by cohesive traction. Therefore, the damage scale $d$ for the cohesive behaviour along the crack can be expressed as below.

$$
d=\frac{\int_{\Omega} \frac{1}{2} t_{0} \varepsilon d \Omega-\int_{\Omega} \frac{1}{2} t \varepsilon_{0} d \Omega}{G_{c} l_{\text {crack }}}
$$

Where, $l_{\text {crack }}$ is the characteristic length of a crack, within which, the damage status was considered to be coincident. When $d=1$ the cohesive traction vanishes, and the cohesive crack evolves to a strong discontinuity (element separated). In Equ. 9, $\mathrm{t}_{0}$ and $\varepsilon_{0}$ are, respectively, the initial traction or cohesive strength and the initial damage strain when the damage starts. Because the softening constitutive law shown in Fig. 2 was used for reducing the cohesive traction, i.e. $t=(1-d) t_{0}$, then we can achieve an explicit expression of the equivalent damage scale as shown below.

$$
d=\frac{\int_{\Omega} \frac{1}{2} t_{0} \varepsilon d \Omega-\int_{\Omega} \frac{1}{2} t_{0} \varepsilon_{0} d \Omega}{G_{c} l_{\text {crack }}-\int_{\Omega} \frac{1}{2} t_{0} \varepsilon_{0} d \Omega}
$$


Thus the nodal force with the existence of cohesive traction at the crack can be expressed as:

$$
\begin{aligned}
& \mathbf{f}_{\text {coh }}^{\mathrm{int}}=\mathbf{K}^{u a}\left(\mathbf{K}^{a a}\right)^{-1} \int_{\Gamma_{\text {con }}} \mathbf{N}^{T} t_{0}(1-d) d \Gamma \\
& =\mathbf{K}^{u a}\left(\mathbf{K}^{a a}\right)^{-1} \int_{\Gamma_{\text {cor }}^{\text {crack }}} \mathbf{N}^{T} t_{0}\left(\frac{G_{c} l_{\text {crack }}-\int_{\Omega} \frac{1}{2} t_{0} \varepsilon d \Omega}{G_{c} l_{\text {crack }}-\int_{\Omega} \frac{1}{2} t_{0} \varepsilon_{0} d \Omega}\right) d \Gamma
\end{aligned}
$$

Substituting Equ. 11 into Equ. 7 results the final condensed discrete form of equilibrium equation as shown in Equ. 12.

$$
\left(\mathbf{K}_{r}^{u u}-\mathbf{K}_{r}^{u a}\left(\mathbf{K}_{r}^{a a}\right)^{-1} \mathbf{K}_{r}^{a u}+\mathbf{K}_{r}^{u a}\left(\mathbf{K}_{r}^{a a}\right)^{-1}\left(\mathbf{L}_{1}-\mathbf{L}_{2}\right)\right) \mathbf{u}=\mathbf{f}_{e x t}^{u}
$$

In Equ. 12, the left hand side within the outer bracket is an equivalent stiffness matrix including the effects from enrichment and cohesive force. The following two symbols were used.

$$
\begin{gathered}
\mathbf{L}_{1}=\int_{\Gamma_{\text {con }}} \mathbf{N}^{T} t_{0} \frac{G_{c} l_{\text {crack }}}{G_{c} l_{\text {crack }}-\int_{\Omega} \frac{1}{2} t_{0} \varepsilon_{0} d \Omega} d \Gamma \mathfrak{R} \\
\mathbf{L}_{2}=\int_{\Gamma_{\text {con }}} \mathbf{N}^{T} t_{0} \frac{\int_{\Omega} \frac{1}{2} t_{0} \varepsilon d \Omega}{G_{c} l_{\text {crack }}-\int_{\Omega} \frac{1}{2} t_{0} \varepsilon_{0} d \Omega} d \Gamma \Re
\end{gathered}
$$

Where, $\mathfrak{R}$ was chosen an operator for obtaining the implicit expression for the ultimate equilibrium and given by Equ. 14 .

$$
\mathfrak{R}=\frac{1}{\left(\mathbf{K}_{r}^{u a}\left(\mathbf{K}_{r}^{a a}\right)^{-1} \int_{\Gamma_{\text {con }}^{\text {crack }}} \mathbf{N}^{T} t_{0} d \Gamma\right)^{T} \mathbf{u}}\left(\mathbf{K}_{r}^{u a}\left(\mathbf{K}_{r}^{a a}\right)^{-1} \int_{\Gamma_{\text {coh }}^{\text {crack }}} \mathbf{N}^{T} t_{0} d \Gamma\right)^{T}
$$

\section{Crack propagation scheme}


It can be seen from Equ. 12 that the standard polynomial shape functions and conventional FEM DoFs are used in the ECDM. However, the effects from enriched degree freedoms and cohesive force were accounted into the equivalent stiffness matrix in the left side of Equ. 12. Through the user subroutine UEL in the commercial code ABAQUS, the ECDM was programmed as a user element. This investigation has considered three damage cases, interlaminar delamination, intralaminar matrix cracking and fiber breakage. Therefore, different damage criterions were employed to characterize various failure mechanisms. For delamination initiation, the quadratic failure criterion [9] was employed.

$$
F(\tau)=\left(\frac{\left\langle\tau_{n}\right\rangle}{N}\right)^{2}+\left(\frac{\tau_{s}}{S}\right)^{2}=1
$$

In which \langle\rangle is the Macauley operator, $\tau_{n}$ and $\tau_{s}$ are the normal traction and shearing traction on interface. $N$ and $S$ are the interlaminar tensile strength and the shear strengths, respectively. This criterion has been successfully used to predict the onset of delamination in author's previous investigations [22-24]. For mix-mode delamination propagation, based on the mix-mode criterion proposed by Benzeggagh and Kenane [25], a total fracture toughness is computed with regard to a function of crack mode ratio:

$$
G_{c}=G_{I, c}+\left(G_{I I, c}-G_{I, c}\right)\left(\frac{1}{1+\beta}\right)^{\eta}
$$

In which the mix-mode parameter $\eta$ was taken as 1.39 in this investigation [25]. For a normal opening delamination case, the mode mixed ration $\beta$ is defined as: 
$\beta=\left|\frac{\varepsilon_{0}^{n} / N}{\varepsilon_{0}^{s} / S}\right|$

Where, $\varepsilon_{0}^{n}$ and $\varepsilon_{0}^{s}$ are respectively the initial normal and shear strain at the onset of material softening under mixed-mode loading. The path of delamination propagation was prescribed along the physical interface boundary.

In the cases for matrix cracking and fibre fracture, a maximum principal stress based criteria was used to characterize the damage propagation, which means when the maximum principal stress $\sigma_{\max }$ at Gauss points of any elements is beyond the cohesive strength of matrix or fibre, the damage occurs. The direction perpendicular to the maximum principal stress was adopted to be the crack direction within elements which have potential arbitrary cracks. Three fracture modes in laminated composites are schematically illustrated in Fig. 3, which gives the presence of three fracture mechanisms, delamination, matrix cracking and fibre breakage. Stresses at the mid-point of the interface within corresponding elements were used for the judgement of delamination propagation, while the average maximum principal stresses at four Gauss points within upper layer $\left(90^{\circ} \mathrm{ply}\right)$ and lower layer $\left(0^{\circ} \mathrm{ply}\right)$ related elements were calculated in the judgements for matrix crack and fibre breakage, respectively. The fracture direction for both matrix crack and fibre breakage was veraciously determined by the direction with $\theta_{\max }$ which is perpendicular to the maximum principal stress. 


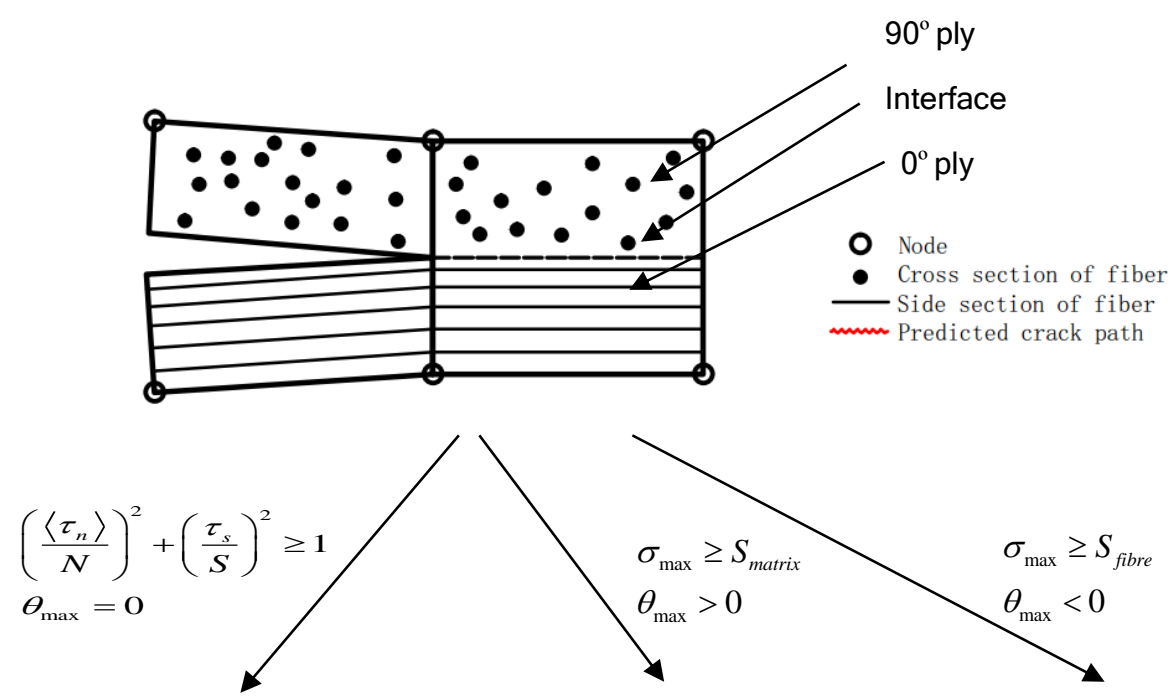

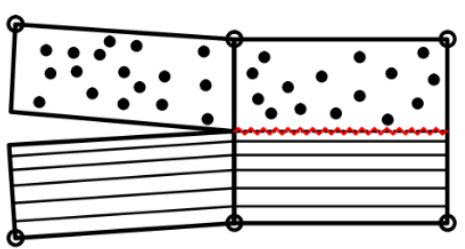

(a) Delamination

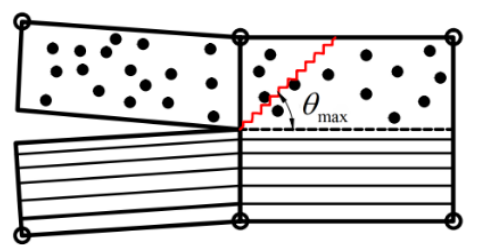

(b) Matrix cracking

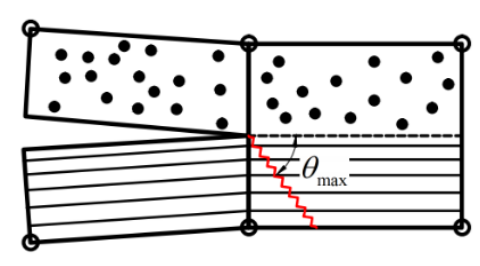

(c) Fibre breakage

Fig. 3 Three schematic failure modes and their initial judgements

\section{Implementation and numerical Integration}

The flow chart of implementation of the ECDM is shown in Fig. 4. In this implementation, similar with standard FEM, it is necessary to perform numerical integration over the element domain to compute the element stiffness matrix. Normally, Gauss method and Simpson' rule are employed as the integration algorism. However, because of the discontinuity the calculation of stiffness matrix of the ECDM user element embracing cracks needs to split the element domain into sub-cells (triangles for example) aligned to the discontinuous surface in which the 
integrands are continuous and differentiable when conducting the numerical integration $[26,27]$.

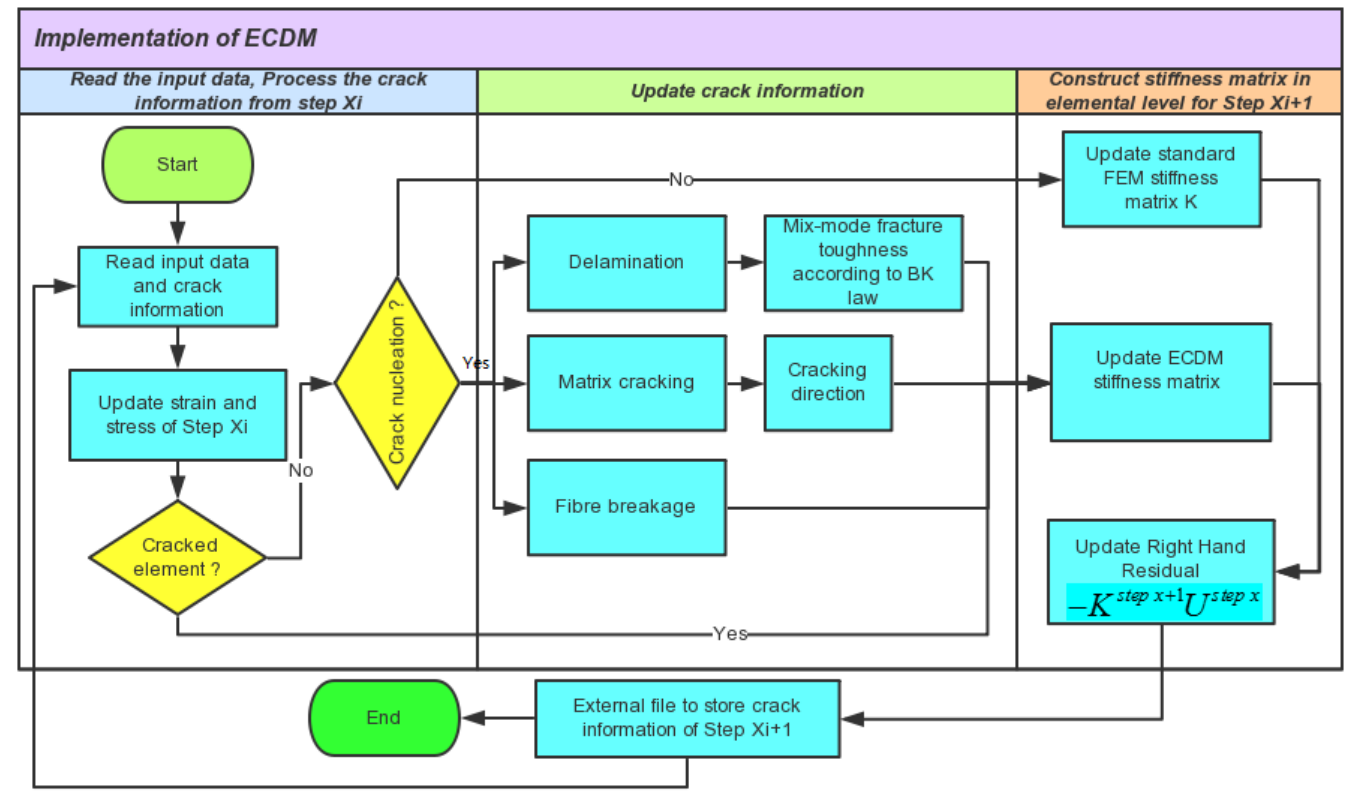

Fig. 4 The flow chart of the ECDM

Fig. 5 shows proposed subdivision schemes used in the ECDM. The purpose of sub-elements dividing is solely for the purpose of numerical integration; it does not introduce new degrees of freedom. Gaussian quadrature was used to perform with the integration points within each triangular or quadrangle sub-element. In the first case, the enhanced element was divided into two quadrangles both of which were treated as a separate element with four gauss points (open circles) during the integration process. In the second case, six sub-triangle domains were prepared for integration. Each sub-triangle domain possesses three integration points (open circles). The integration points (solid circles) on the discontinuity curve are for the purpose of computing the cohesive traction between the crack surfaces. 


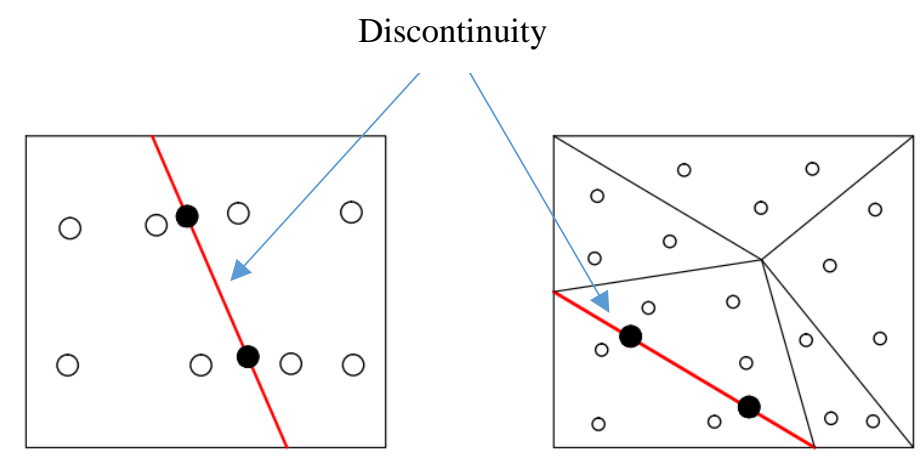

Fig. 5 Two schemes for intersecting element by discontinuity curve. (a) dividing a quadrilateral into two sub-quadrilaterals; (b) dividing a quadrilateral into six triangles.

The element stiffness matrix can be obtained by superposition of integration results from all sub-elements as shown in Equ. (18).

$$
K_{\text {element }}=\sum_{i=1}^{n} K_{\text {subelement }}^{i}
$$

Newton-Raphson method with line search scheme was employed for the non-linear iteration in the ECDM based FE analysis. In order to improve the iteration convergence in the post-softening regime, the viscous regularization scheme [28] was introduced which can speed up the simulation.

\section{Numerical applications}

\subsection{Stiffened laminated composite panel}

The first example is a stiffened laminated composite panel under four-point bending. The dimension and lay-up for the host panel or skin and the stiffener are shown in Fig 6. The skin consists of 32 unidirectional plies and the stiffener has 24 plies, each layer 
is $0.15 \mathrm{~mm}$ thick. There is a $0.2 \mathrm{~mm}$ adhesive layer between the skin and the stiffener,

i.e. the stiffener is bonded onto the skin. The mechanical properties of unidirectional lamina and adhesive are given in Table 1 . Considering the symmetric and boundary conditions of the panel, a half FE mesh was employed in the modelling analysis.

Table 1 Material properties of stiffened fibre composite panel

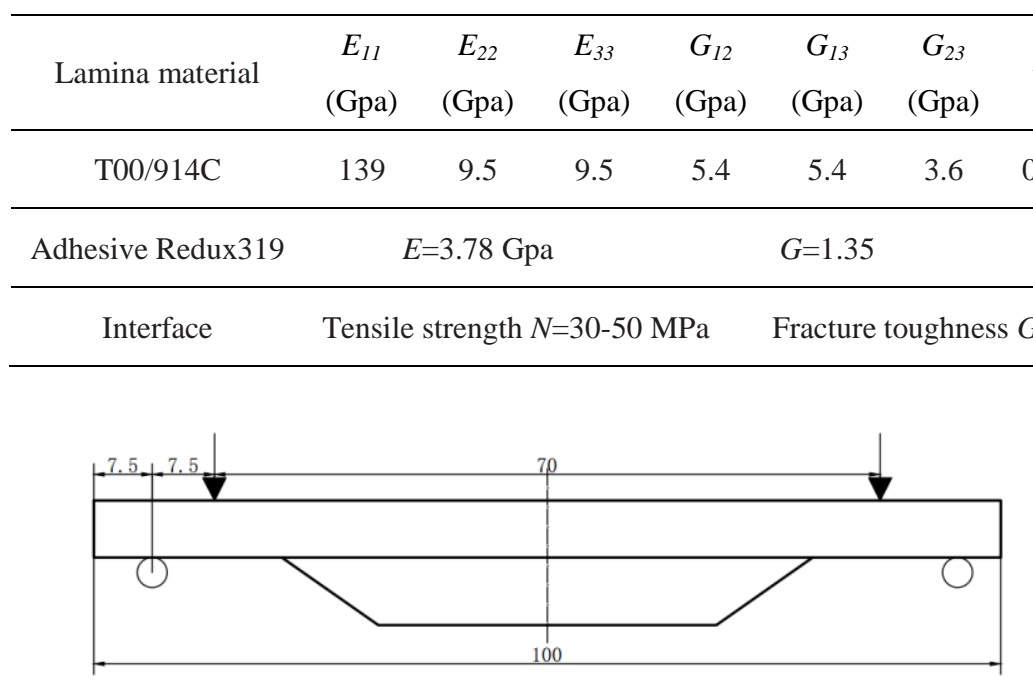

Skin lay-up:

$\left[(45 /-45 / 0 / 90)_{4}\right]_{\mathrm{s}}$

Stiffener lay-up:

$\left[(45 /-45 / 0)_{2 s}\right]_{s}$

Fig. 6 Configuration of the stiffened fibre-composite panel
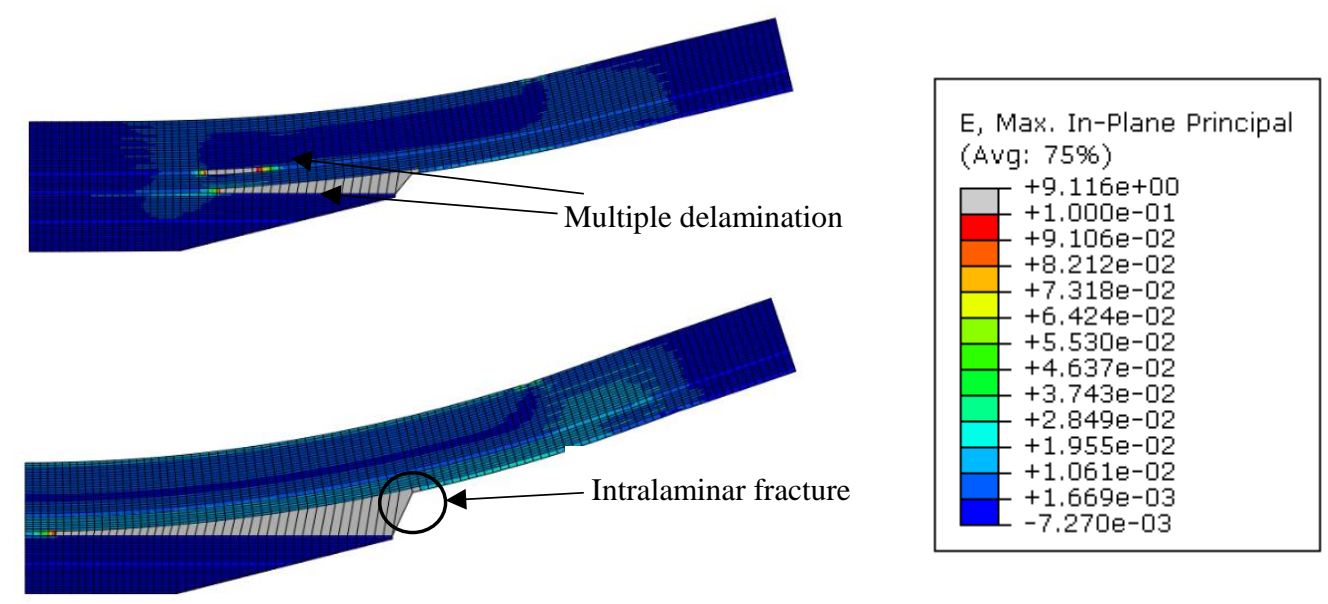

Fig. 7 a. Multiple layered delamination; b. Intralaminar failure and final delamination 


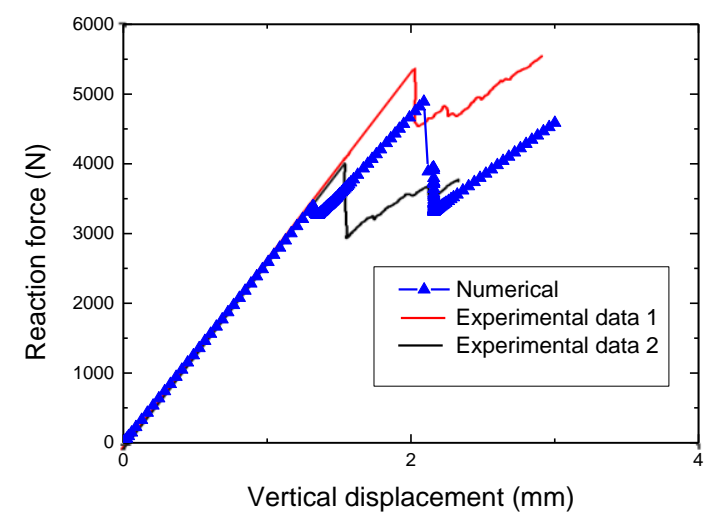

Fig. 8 Load-displacement curves given by the ECDM and experimental work

Fig. 7 shows multicrack failure patterns, which are identical to the observations of corresponding experimental work [22]. Fig. 7a shows multiple layered delamination, while Fig. 7b shows intralaminar fracture including fibre breakage as it is at the $45^{\circ}$ ply and the final delamination. Firstly, delamiantion initiated at the corner close to the end of the stiffener, propagated towards to the middle of the panel along the interface one layer above the bonding interface between skin and stiffener, which is between the first $\left(+45^{\circ}\right)$ and second $\left(-45^{\circ}\right)$ ply. Meanwhile, there was a minor delamination occurred at the interface between another $45^{\circ}$ and $-45^{\circ}$ interface seven layers above the first $45^{\circ}$ and $-45^{0}$ interface. When load was increased, an intralaminar failure occurred in the $45^{\circ}$ ply at the end of the stiffener. Because of this intralaminar failure the major delamination went through the first $45^{\circ}$ and $-45^{\circ}$ interface and totally separated the stiffener from the skin. In this modelling simulation, when major delamination went through interface, the minor delamination seems closed due to the significant bending but actually it was a delamination. 
The comparison of failure response between the modelling prediction and two experimental measurements is shown in Fig. 8. It can be seen from Fig. 8 that there is a gap of the tested failure load between two samples. The one with lower strength is possibly because of pre-existing defects from specimen manufacture. In general, modelling prediction has good agreement with experiment results regarding initial stiffness, failure load and residual stiffness. The experimentally measured failure response roughly recorded the failure load regarding the final delamination presented by a response drop shown in Fig. 8. In the ECDM predicted failure response shown in Fig. 8, there is a small or first response drop regarding the early multiple layered delamination shown in Fig. 7a, which was not captured by the experiment measurement. When the displacement was increased to $2.05 \mathrm{~mm}$, an intralaminar failure with fibre breakage occurred at the $45^{\circ}$ ply, and immediately followed by the final delamination in which the panel was split. These two major cracks were reflected by a predicted significant or second response drop shown in Fig. 8. As long as the fibre breakage at the $45^{\circ}$ ply and the final delamination happen, the global bending stiffness displayed a significant degradation. Simultaneously the relatively high strain field within the upper delamination region was released when the final delamination went through the panel. The rest stiffness is the residual stiffness from the skin only. In this modelling investigation, the ECDM reproduced the multicrack evolution in the laminated stiffened panel, which has confirmed that the proposed 
numerical model is an appropriate approach in simulating the multicrack failure including multiple layered delamination, intralaminar failure and fibre breakage.

\subsection{Laminated composite $\mathrm{T}$-joint}

Fibre composite T-joint component, as a crucial connection, normally can be found in many engineering structures such as aircraft, shipbuilding, aero engine blade, wind turbine blade, etc. Because of its relative complexity of configuration, predicting both damage resistance and failure mechanism is one of major concern in the design of composite structures which contain T-joints. Indeed, the dramatic change in geometry and discontinuity of the fibre reinforcements in the structure makes the T-joint a potentially weak point affecting the overall efficiency and integrity of the structures. Study of the failure mechanism of the T-joint specimen under service loading conditions has been carried out via conventional cohesive damage model (CDM) [22-24]. However, attributing the complicated geometrical configuration of T-joint specimen, the meshing work in the CDM based modelling is cumbersome. In addition, to be able to meet the purpose of describing the multiple layered delamination, the interface cohesive elements are required to be inserted into each layer, which would course a convergent failure problem in nonlinear iteration. Thus the single major delamination was modelled in most previous modelling work.

Herein, the multiple layered delamination of T-joint under pulling and bending was modelled using the proposed ECDM. The geometry of T-joint specimen was taken 
from the reference [22]. Fig. 9a shows the loading condition with bending and pulling plus clamped restraints on the foot of T-joint. Fig. 9b shows the lay-up configuration in the deltoid region of $\mathrm{T}$-joint. The properties of the material constitutes of the T-joint component is summarized in Table 2.
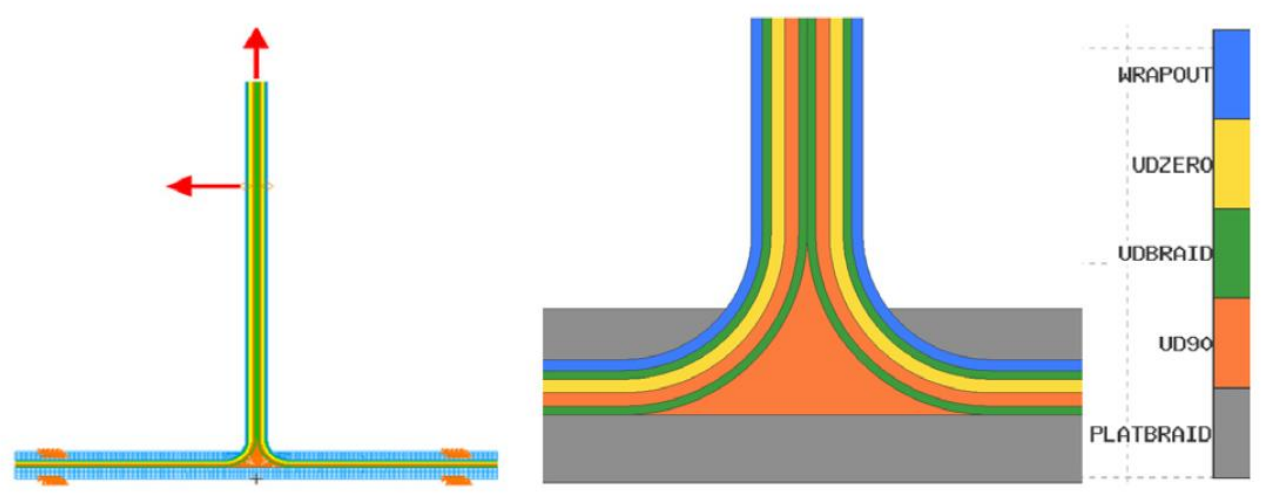

Fig. 9 a. A model of T-joint under bending and pulling; b. Lay-up configuration of T-joint

Table 2 Material properties

\begin{tabular}{cccccccccc}
\hline Material constitute & $\begin{array}{c}E_{11} \\
(\mathrm{Gpa})\end{array}$ & $\begin{array}{c}E_{22} \\
(\mathrm{Gpa})\end{array}$ & $\begin{array}{c}E_{33} \\
(\mathrm{Gpa})\end{array}$ & $\begin{array}{c}G_{12} \\
(\mathrm{Gpa})\end{array}$ & $\begin{array}{c}G_{13} \\
(\mathrm{Gpa})\end{array}$ & $\begin{array}{c}G_{23} \\
(\mathrm{Gpa})\end{array}$ & $v_{12}$ & $v_{13}$ & $v_{23}$ \\
\hline Outer braided wrap & 59.7 & 60.1 & 9.7 & 21.9 & 4.7 & 4.7 & 0.27 & 0.28 & 0.28 \\
Braided UD layer & 160 & 9.7 & 9.7 & 5.9 & 5.9 & 4.7 & 0.33 & 0.33 & 0.28 \\
{$\left[0^{\circ}\right]$ layer } & 152 & 9.7 & 9.7 & 5.9 & 5.9 & 4.7 & 0.33 & 0.33 & 0.28 \\
{$\left[90^{\circ}\right]$ layer and deltoid } & 9.7 & 152.0 & 9.7 & 5.9 & 4.7 & 5.9 & 0.02 & 0.28 & 0.33 \\
Platform braids & 65.8 & 46.1 & 9.7 & 25.8 & 4.7 & 4.7 & 0.42 & 0.28 & 0.28 \\
Interface & & $N_{t}=45 \mathrm{Mpa}$, & $N_{s}=35 \mathrm{Mpa}$, & $G_{I c}=0.3 \mathrm{~kJ} / \mathrm{m}^{2}$, & $G_{I I c}=1.0 \mathrm{~kJ} / \mathrm{m}^{2}$ & \\
\hline
\end{tabular}

Fig. 10 shows experimentally observed failure mechanisms in the area of deltoid region of T-joint under (a) bending and (b) pulling, respectively. Figs. 11 and 12 show the ECDM predicted two failure stages presented by maximum principal strain 
contours, initial crack and final fracture, with multicrack failure including multiple layered delamination and intralaminar crack in both bending and pulling cases. In general, the predicted multicrack failure has good agreement with experimental observation in both bending and pulling cases. The failure pattern coursed by bending is dissymmetric delamination and typical multi-delamination distributed at different interfaces in the deltoid region of $\mathrm{T}$-joint as shown in Fig. 11. The multi-delaminations predicted in the right part of radius laminates basically agree with experimental observation as shown in Fig. 10. Fig. 11a shows the initial crack stage with corresponding load $15 \mathrm{~N} / \mathrm{mm}$ : a delamination between deltoid and UD braid layer started at the tension side of the deltoid, followed by a matric crack in the upper part of the deltoid, and another delamination occurred at the compression side of the deltoid. Fig. $11 \mathrm{~b}$ presents the final fracture stage when load increased to $27.6 \mathrm{~N} / \mathrm{mm}$ : the delamination at tension side of the deltoid developed further from the initial crack stage and totally separated the web from the platform, meanwhile, another delamination occurred at the UD $90^{\circ}$-UD $0^{0}$ interface in the web region.

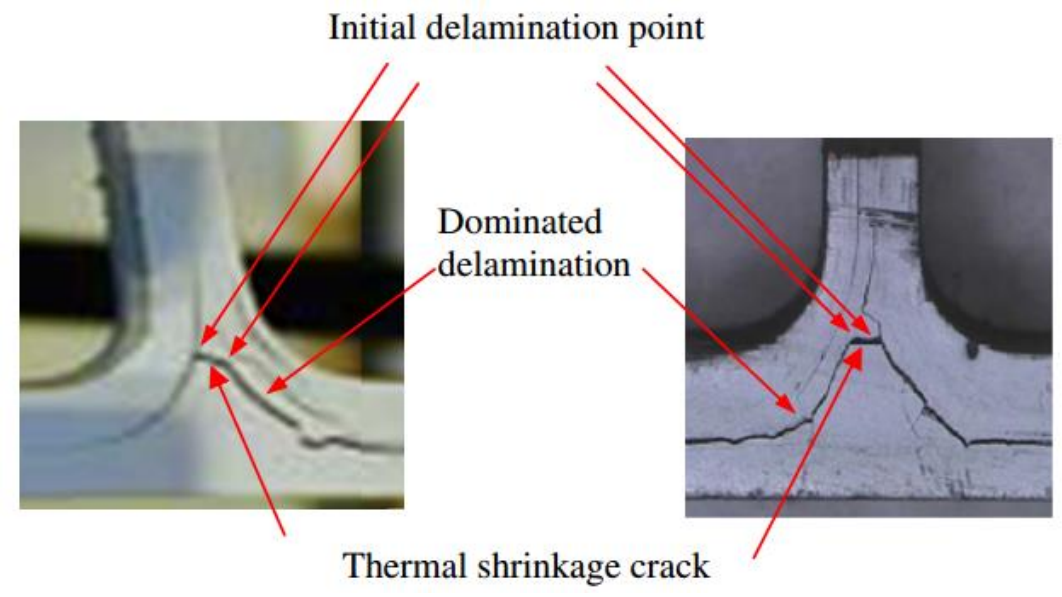

Fig. 10 Failure pattern of T-joint under (a) bending and (b) pulling 

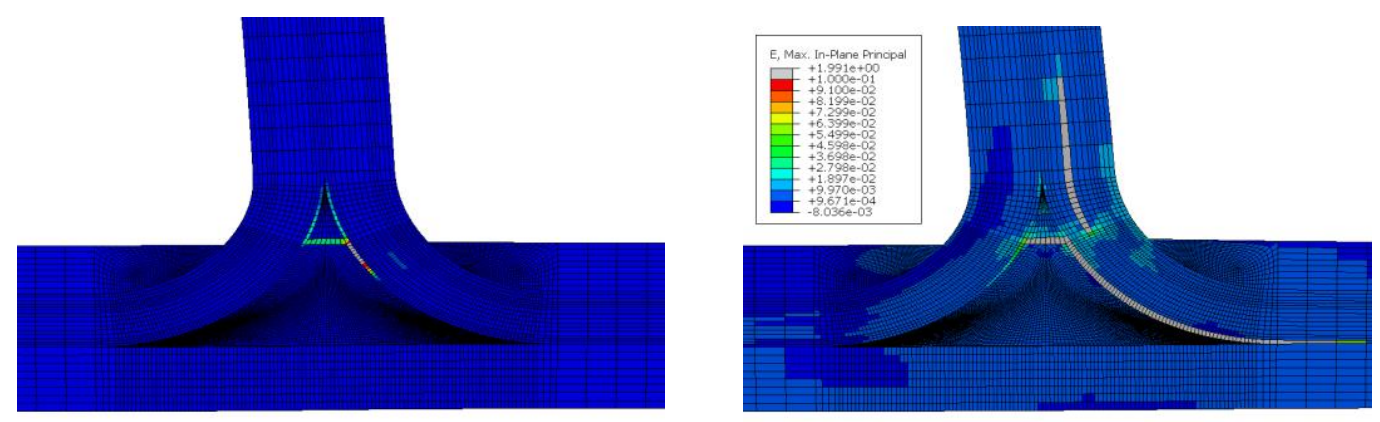

Fig. 11 Crack propagation of T-joint under bending, (a) initial crack; (b) final fracture

Considering the symmetry of the T-joint, a half mesh was modelled for pulling case, the predicted delamination in deltoid region of T-joint is shown in Fig. 12. Fig 12a shows initial crack stage when load increased to $122 \mathrm{~N} / \mathrm{mm}$ : a delamination propagated from the middle of radius laminate upward and downward along the interface between deltoid and UD braid layer. Fig. 12b presents the final fracture stage with corresponding load $150 \mathrm{~N} / \mathrm{mm}$ : the delamination from initial crack stage developed further and totally separated the web from the platform of T-joint, meanwhile a matric crack occurred at the upper part of the deltoid region.
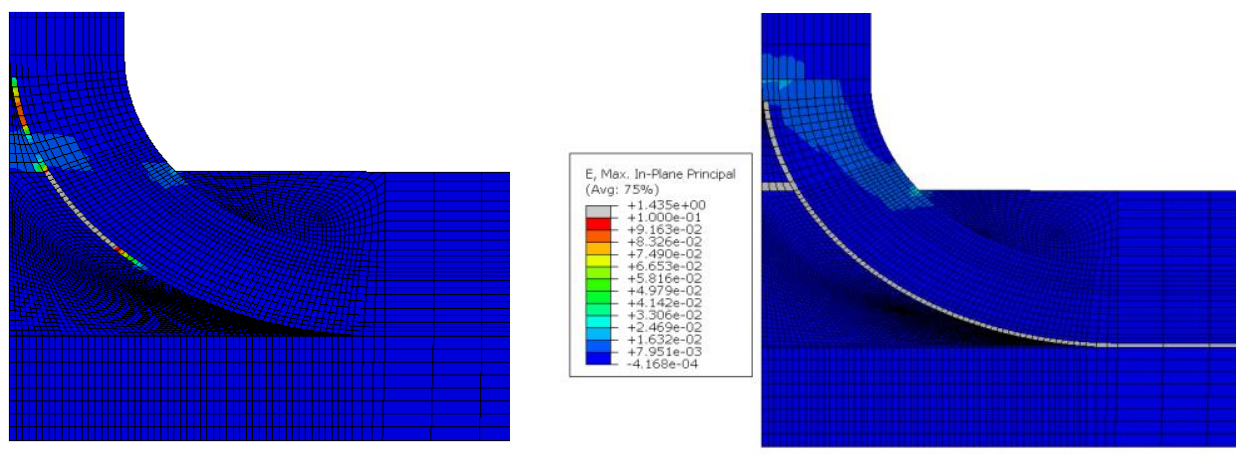

Fig. 12 Crack propagation of T-joint under pulling, (a) initial crack; (b) final fracture

Figs. 13a and 13b show the ECDM predicted load-displacement curves in bending and pulling case. It can be seen from Figs.13a and 13b that there are two response 
drops in load-displacement curves in both bending and pulling cases. The first drop reflects the initial crack while the second drop reflects the final fracture shown in Figs. 11 and 12. Although there is no experimentally measured load-displacement curve reported by previous work regarding this T-joint investigation [22-24], comparison of the failure loads between predictions and tests are shown in Fig. 14 in which a good agreement between prediction and test can be observed. Actually, the tested mean of failure load $33.5 \mathrm{~N} / \mathrm{mm}$ in bending case is associated with the predicted failure load $27.6 \mathrm{~N} / \mathrm{mm}$ at the modelled final fracture with a significant reduction of stiffness; while the experimentally measured failure load $113 \mathrm{~N} / \mathrm{mm}$ in pulling case is associated with the predicted failure load $122 \mathrm{~N} / \mathrm{mm}$ at the modelled initial crack stage with a big response drop; the error in both cases is less than $8 \%$. This ECDM based modelling demonstrated a reasonable prediction comparing to experimentally measured failure load, and disclosed detailed multicrack mechanism in this composite T-joint, which is not easy to be observed in experimental work.
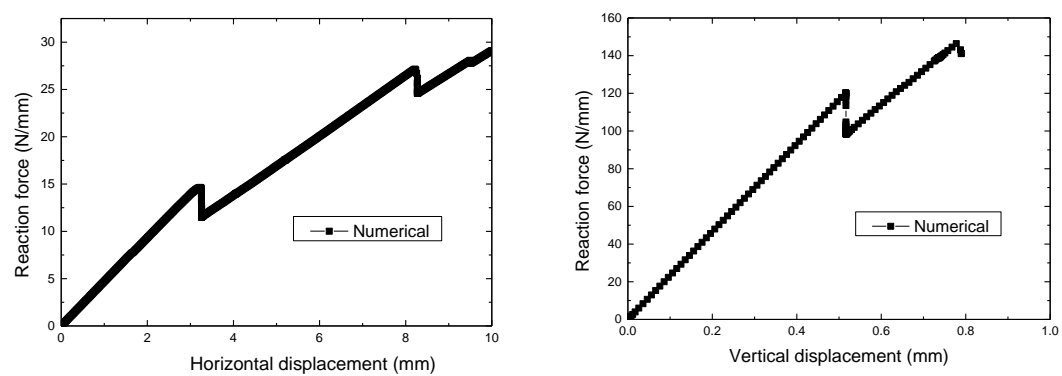

Fig. 13 The ECDM predicted load-displace curves of T-joint under (a) bending and (b) pulling 


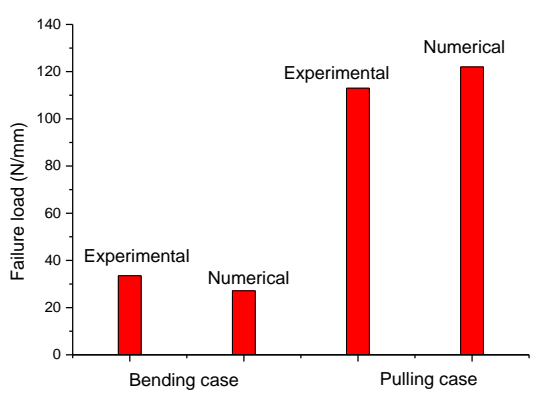

Fig. 14 Comparison of failure loads between numerical predictions and average tested results

\section{Conclusion and future work}

The proposed novel ECDM was successfully implemented as a user element using the subroutine UEL of commercial FE package ABAQUS to investigate multicrack evolution in laminated composites. Examples given in this investigation verified the capacity of the ECDM in prediction of multicrack evolution including multiple layered delamination, matrix crack and fibre breakage. Because the ECDM does not employ additional enriched degree freedoms, and pre-prepared crack path is no longer required, the ECDM is an efficient modelling approach in studying multicrack failure mechanism in laminated composites. Considering the length of this paper, detailed investigation of efficiency of the ECDM will be discussed by different papers. It can be concluded that the ECDM is capable of capturing the complex multicrack evolution in composite structures without prior knowledge for the crack location and propagation direction. With this tool, a comprehensive numerical prediction of failure response of composite structures can be accessed with less computational endeavour. In the future work, the ECDM will be applied in three dimensional models to predict multicrack evolution in three dimensional solid composite structures. 


\section{Reference}

[1] Kachanov LM. Time of rupture process under creep conditions. Izy Akad Nank S.S.R. Otd Tech Nauk 1958;8:26-31.

[2] Rabotnov YN. Creep rupture. In: Proceedings of the XII international congress applied mechanics. Stanford: Springer; 1968

[3] Naghipour, P., K. Schulze, J. Hausmann, and M. Bartsch. "Numerical and experimental investigation on lap shear fracture of Al/CFRP laminates."Composites Science and Technology 72, no. 14 (2012): 1718-1724.

[4] Melro, A. R., Camanho, P. P., Pires, F. A., \& Pinho, S. T. (2013). Micromechanical analysis of polymer composites reinforced by unidirectional fibres: Part I-Constitutive modelling. International Journal of Solids and Structures, 50(11), 1897-1905.

[5] Melro, A. R., P. P. Camanho, FM Andrade Pires, and S. T. Pinho. "Micromechanical analysis of polymer composites reinforced by unidirectional fibres: Part I-Constitutive modelling." International Journal of Solids and Structures 50, no. 11 (2013): 1897-1905.

[6] Kim, E. H., Rim, M. S., Lee, I., \& Hwang, T. K. (2013). Composite damage model based on continuum damage mechanics and low velocity impact analysis of composite plates. Composite Structures, 95, 123-134.

[7] Gupta, A. K., Patel, B. P., \& Nath, Y. (2013). Continuum damage modeling of composite laminated plates using higher order theory. Composite Structures, 99, 141-151.

[8] Rinderknecht, S. and Kro"plin, B. (1994). Calculation of Delamination Growth With Fracture and Damage Mechanics, Recent Developments in Finite Element Analysis, CIMNE, Barcelona, Spain.

[9] Chen, M. Crisfield, AJ Kinloch, EP Busso, FL Matthews, Y. Qiu, J. "Predicting progressive delamination of composite material specimens via interface elements." Mechanics of composite materials and structures 6, no. 4 (1999): 301-317.

[10]Liljedahl, C. D. M., A. D. Crocombe, M. A. Wahab, and I. A. Ashcroft. "Modelling the environmental degradation of adhesively bonded aluminium and composite joints using a CZM approach." International Journal of Adhesion and Adhesives27, no. 6 (2007): 505-518.

[11] Ghasemnejad, Hessammaddin, and Akbar Aboutorabi. "Cohesive zone modeling $(\mathrm{CZM})$ in prediction of delamination failure in laminated composite structures." Journal of Materials Science and Engineering 5, no. 1 (2011): 61-64.

[12]Chen, Jiye, and Dominic Fox. "Numerical investigation into multi-delamination failure of composite T-piece specimens under mixed mode loading using a modified cohesive model." Composite Structures 94, no. 6 (2012): 2010-2016.

[13] Moës, N., \& Belytschko, T., Extended finite element method for cohesive crack growth. Engineering fracture mechanics 2002; 69(7), 813-833.

[14]Mergheim, J., Kuhl, E., \& Steinmann, P., A finite element method for the computational modelling of cohesive cracks. International Journal for Numerical Methods in Engineering 2005; 63(2), 276-289.

[15]Fries, T. P., A corrected XFEM approximation without problems in blending elements. International Journal for Numerical Methods in Engineering 2008; 75(5), 503-532.

[16]Laborde, P., Pommier, J., Renard, Y., \& Salaün, M., High-order extended finite element method for cracked domains. International Journal for Numerical 
Methods in Engineering 2005; 64(3), 354-381.

[17]Chessa, J., Wang, H., \& Belytschko, T., On the construction of blending elements for local partition of unity enriched finite elements. International Journal for Numerical Methods in Engineering 2003; 57(7), 1015-1038.

[18]Tarancón, J. E., Vercher, A., Giner, E., \& Fuenmayor, F. J., Enhanced blending elements for XFEM applied to linear elastic fracture mechanics. International Journal for Numerical Methods in Engineering 2009; 77(1), 126-148.

[19]Xiao, Q. Z., \& Karihaloo, B. L., Improving the accuracy of XFEM crack tip fields using higher order quadrature and statically admissible stress recovery. International Journal for Numerical Methods in Engineering 2006; 66(9), 1378-1410.

[20]Fries, T. P., \& Belytschko, T., The extended/generalized finite element method: an overview of the method and its applications. International Journal for Numerical Methods in Engineering 2010; 84(3), 253-304.

[21] Abaqus User Subroutines Reference Manual. V 6.12. ABAQUS, Inc. Providence, RI.

[22]Chen, J., Ravey, E., Hallett, S., Wisnom, M., \& Grassi, M. (2009). Prediction of delamination in braided composite T-piece specimens. Composites Science and Technology, 69(14), 2363-2367.

[23]Chen, J., \& Fox, D. (2012). Numerical investigation into multi-delamination failure of composite T-piece specimens under mixed mode loading using a modified cohesive model. Composite Structures, 94(6), 2010-2016.

[24]Chen, J. (2012). A numerical investigation of thermal-related matrix shrinkage crack and delamination in composite T-piece specimens using a modified interface cohesive model. Journal of Thermoplastic Composite Materials, 25(3), 267-282.

[25]Benzeggagh, M. L., \& Kenane, M. (1996). Measurement of mixed-mode delamination fracture toughness of unidirectional glass/epoxy composites with mixed-mode bending apparatus. Composites science and technology, 56(4), 439-449.

[26]T. Belytschko and T. Black. Elastic crack growth in finite elements with minimal remeshing. International Journal for Numerical Methods in Engineering, 45:601620, 1999.

[27]S. Bordas, V. P. Nguyen, C. Dunant, A. Guidoum, and H. Nguyen-Dang. An extended finite element library. International Journal for Numerical Methods in Engineering, 71:703-732, 2007.

[28]Duvaut G, Lions JL. Inequalities in mechanics and physics. Berlin: Springer; 1976.

[29]J. Chen, An extended cohesive damage model with a length scale in fracture analysis of adhesively bonded joints, Engineering Fracture Mechanics 2014; 119, 202-213. 